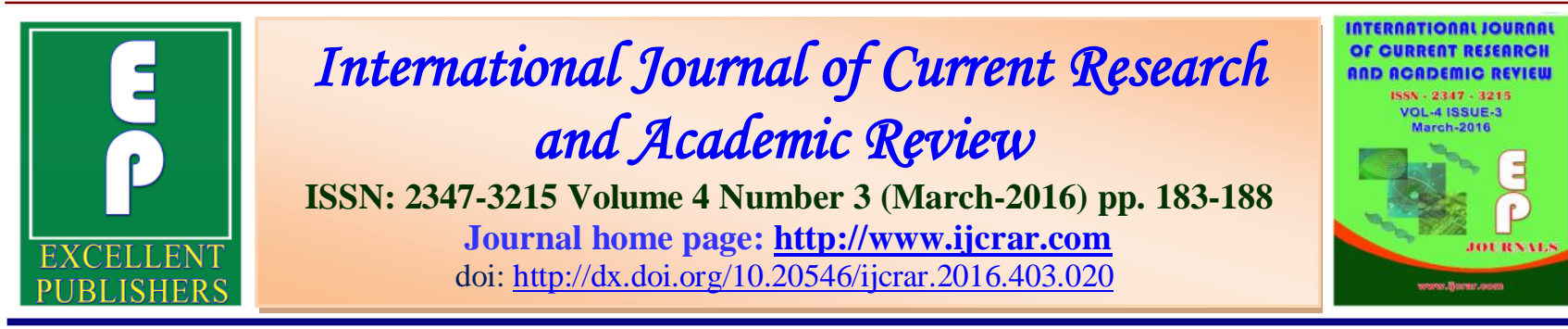

\title{
Case Series of Pseudomonas stutzeri at a Tertiary Care Centre from Kancheepuram District, India
}

\author{
S. Prasanna*, Sunilkumar Jada and Sathya Raj \\ Department of Microbiology, Shri Sathya Sai Medical College and Research Institute, \\ Ammapettai, Kancheepuram-608103, Tamilnadu, India
}

*Corresponding author

\begin{tabular}{|c|c|}
\hline KEYWORDS & $A B S T R A C T$ \\
\hline $\begin{array}{l}\text { Pseudomonas stutzeri, } \\
\text { non-fermenter, } \\
\text { wound infection, } \\
\text { pneumonia, } \\
\text { sinus }\end{array}$ & $\begin{array}{l}\text { Pseudomonas stutzeri is an aerobic, gram negative, oxidase positive non- } \\
\text { fermenter bacillus. P.stutzeri is rarely associated with infections and } \\
\text { infrequently isolated from clinical samples. The main aim of this case series } \\
\text { of four is to highlight the importance of P.stutzeri and the chances of missing } \\
\text { out this organism in reporting due to its colony morphology which look like } \\
\text { aerobic spore bearing organism. The case series are post-operative wound } \\
\text { infection, sinus L4-L5, hospital acquired and community acquired } \\
\text { pneumonia. }\end{array}$ \\
\hline
\end{tabular}

\section{Introduction}

Pseudomonas stutzeri is an aerobic, gram negative, oxidase positive non-fermenter bacillus. It is a ubiquitous organism found in water source, soil and hospital environments $(1,2)$. The organism has been isolated rarely from blood, urine, pus or wound swab, sputum, ear discharge, synovial fluid and hospital equipment.

The first infection by P.stutzeri of non-union fracture tibia was first reported in 1973 by Gilardi et al. The predisposing factors for P.stutzeri infection are immunocompromised patients, patients with underlying illness like chronic renal and liver failure, previous trauma, previous surgeries with nosocomial acquisition, patients on broad spectrum antibiotics and steroids $(1,2,3,4)$.

\section{Case Series Description}

Totally in a period of one month we isolated Pseudomonas stutzeri from four clinical samples. They are post-operative wound infection, sinus $\mathrm{L}_{4}-\mathrm{L}_{5}$, hospital acquired and community acquired pneumonia. 


\section{Case 1}

A 55 year old male presented to general surgery OPD with abdominal pain and swelling over lower abdomen for 1 month duration. The patient was a known case of diabetes and on oral hypoglycaemic drugs. On examination the patient was diagnosed as indirect inguinal hernia over right side, admitted and all pre-operative screening investigations like $\mathrm{HBsAg}, \mathrm{HCV}, \mathrm{HIV}$ antibodies and general routine complete blood counts were done. HBsAg, HCV, HIV were negative and complete blood counts were within normal limit and blood sugar level was also in control. So patient posted for herniorrhaphy and procedure was done successfully. After surgery day 1 and 2 was uneventful, on day 3 there was mild wound gapping with discharge over the operated site. Sutures were removed and wound swab was sent for culture and antibiotic susceptibility testing. Pseudomonas stutzeri was isolated from wound swab and sensitive to almost all antibiotics tested.

\section{Case 2}

A 32 year old non-diabetic male presented to Orthopaedic OPD with back pain and discharge over lower back for 3 weeks duration. On complete clinical examination patient was having discharging sinus over L4-L5 region and suspected as Pott's spine. The wound swab and discharge was sent for mycobacterial and bacterial culture and sensitivity. On processing Ziehl-Neelsen stain- no AFB seen and Gram stainnumerous pus cells with gram negative bacilli was seen. On culture, no growth on LJ medium after 8 weeks and P.stutzeri was isolated from blood agar, MacConkey agar and sensitive to almost all antibiotics tested.

\section{Case 3}

A 60 year old male presented to general medicine OPD with breathlessness and expectorating cough for 2 weeks duration. The patient was a known case of diabetes and on oral hypoglycaemic drugs. On clinical examination of the patient, respiratory system- wheeze with crackles, other systems are within normal limit. The patient's was diagnosed as acute bronchitis with secondary pneumonia, admitted and sputum sample sent for culture, gram stain and acid fast stain and other routine complete blood counts also done. All blood investigations are within normal range except blood sugars, the patient was started with empirical antibiotics and insulin. The sputum sample ZN stain - no AFB seen and gram stain showed numerous pus cells with gram negative bacilli. P.stutzeri was isolated from sputum culture and sensitive to almost all antibiotics tested.

\section{Case 4}

A 42 year old non-diabetic female, she was known hypertensive and CVA with left hemiparesis presented to general medicine OPD with inability to use her right hand and leg for 3 months duration. She was admitted for the presenting complaints and routine complete blood count, blood sugar level and renal and liver function tests were done. All investigations are within normal limits except she was diagnosed to have abnormal renal function tests. The patient was managed with anti-hypertensives, diuretics and physiotherapy. After 3 days of treatment patient developed breathlessness with severe wheeze and crackles, the patient was shifted to medical intensive care unit and diagnosed as pneumonia. The patient was maintained on nasogastric tube for feeding and started with empirical antibiotics covering gram positive and gram negative bacteria. The rest of the treatment was continued and sputum sample was sent for bacteriological examination and culture. The sputum $\mathrm{ZN}$ stain showed no AFB and gram stain 
showed numerous pus cells with gram negative bacilli. P.stutzeri was isolated from sputum culture and sensitive to almost all antibiotics tested.

The colony morphology, biochemical characters and antibiotic susceptibility profile are discussed for all samples together. The samples were processed in blood agar, MacConkey agar and chocolate agar. Growth on blood agar showed 2-3 mm, grey to yellow coloured, raised, dry, wrinkled colonies with regular margin and no hemolysis. Growth on MacConkey agar showed 2-3 mm, raised, dry and wrinkled non-lactose fermenting colonies with regular margin. The Gram stain from the colony showed gram negative bacilli and catalase positive, oxidase positive and motile. The other biochemical reactions: Oxidative utilization of glucose and maltose in HughLeifsen/OF medium, reduces nitrate to nitrite with gas, citrate utilized, urea not hydrolysed, lysine and arginine decarboxylase was negative, non-fermenter with no $\mathrm{H}_{2} \mathrm{~S}$ in TSI media, indole negative. The antibiotic susceptibility testing was carried out on Mueller Hinton agar with 0.5 McFarland's standard suspension of the colonies with following antibiotics: imipenem, gentamicin, amikacin, ciprofloxacin, Cefepime, Ceftazidime, Cefotaxime, Colistin and cotrimoxazole. The organism was identified as Pseudomonas stutzeri with the help of colony characters, biochemical reactions and sensitive to all the above antibiotics except cotrimoxazole.

Fig 1: A-Colony morphology of P.stutzeri in blood agar showing2-3 $\mathrm{mm}$, grey to yellow coloured, raised, ruff, wrinkled colonies with regular margin and no hemolysis; B- Colony morphology of P.stutzeriin MacConkey agar showing 2-3 mm, raised, ruff, wrinkled non-lactose fermenting colonies with regular margin.

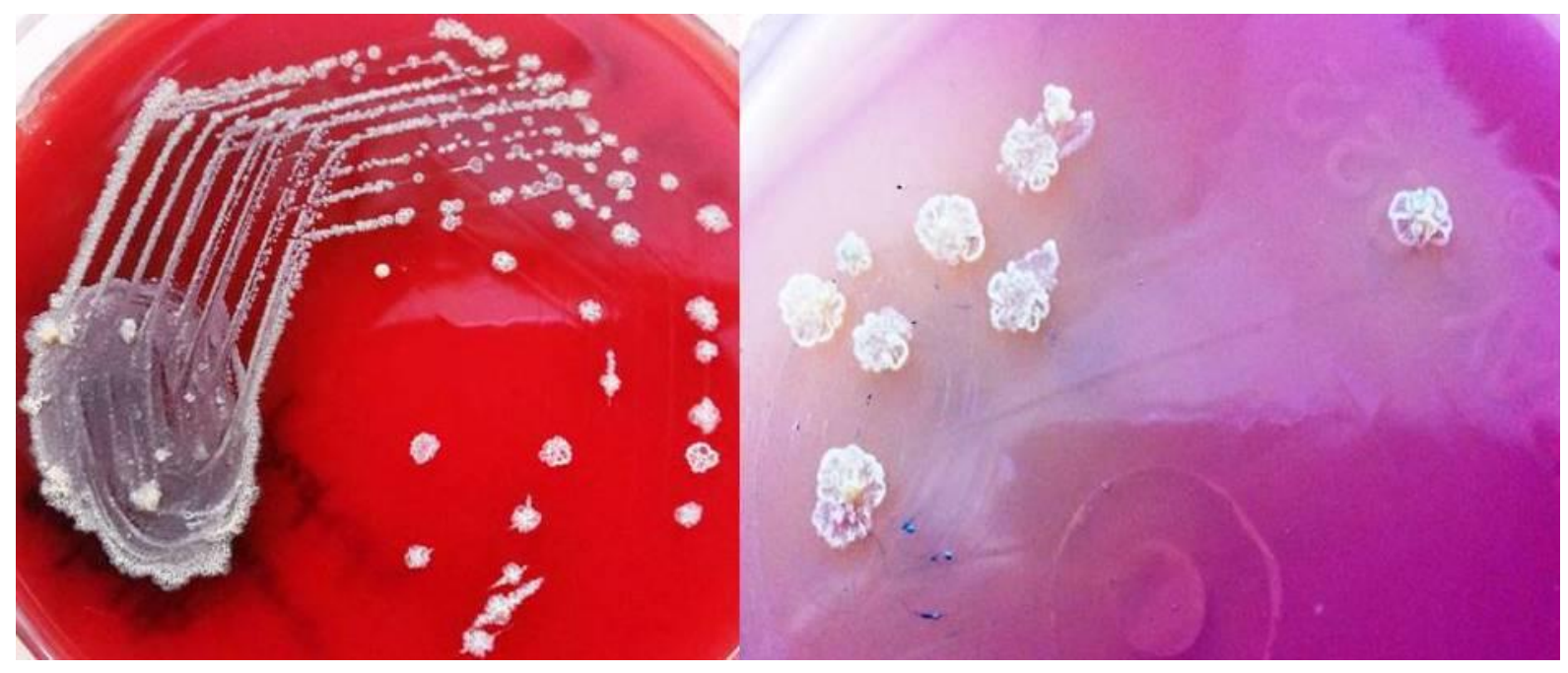


Fig.2 A- Indole negative, urea not hydrolysed, citrate utilized, Non-fermenter without $\mathrm{H}_{2} \mathrm{~S}$ in TSI and motile in mannitol motility medium; B- Lysine and arginine decarboxylase negative.

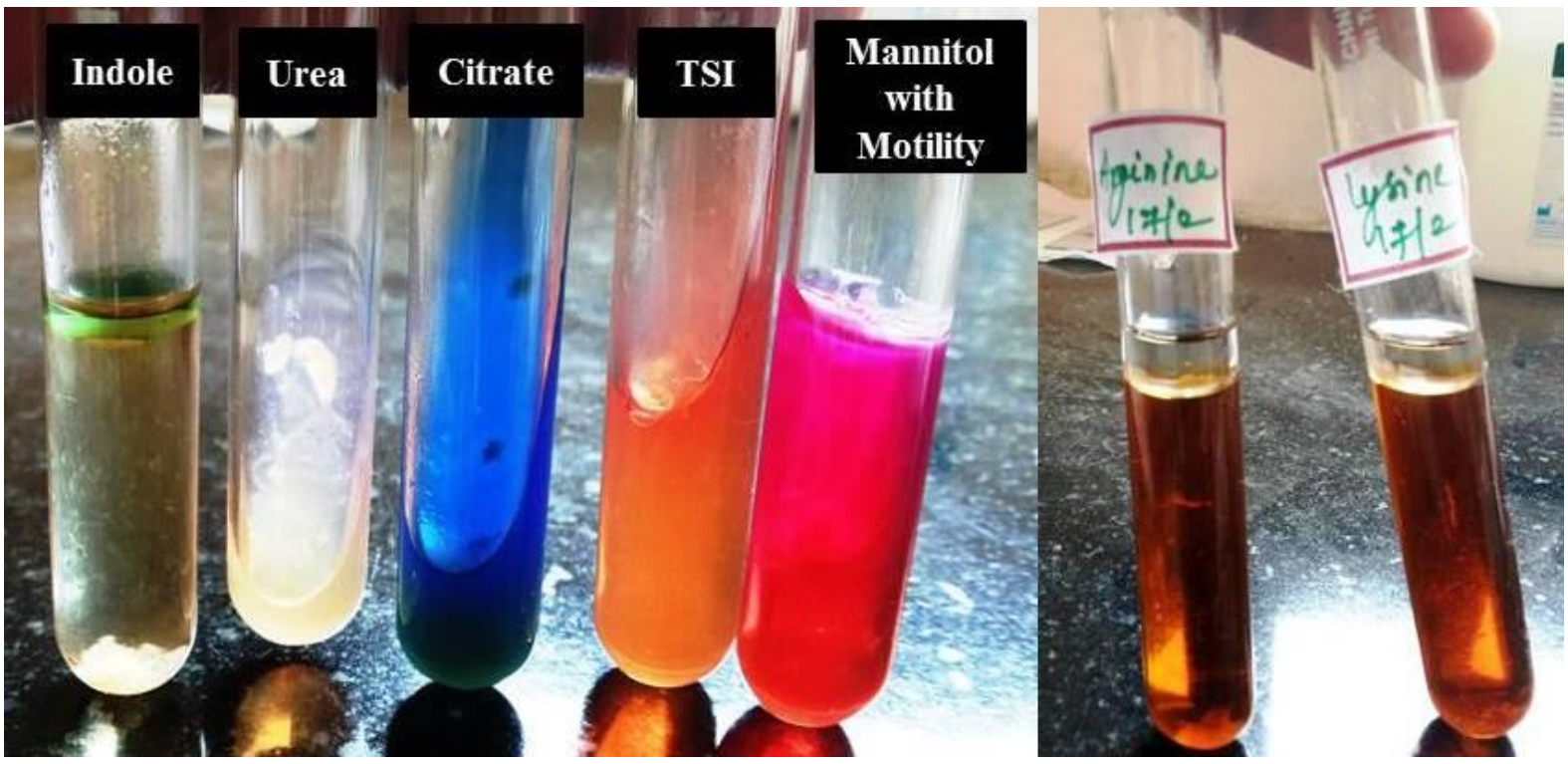

Fig.3 A- Oxidative utilization of glucose in Hugh-Leifsen/OF medium; B- Oxidative utilization of maltose in Hugh-Leifsen/OF medium.

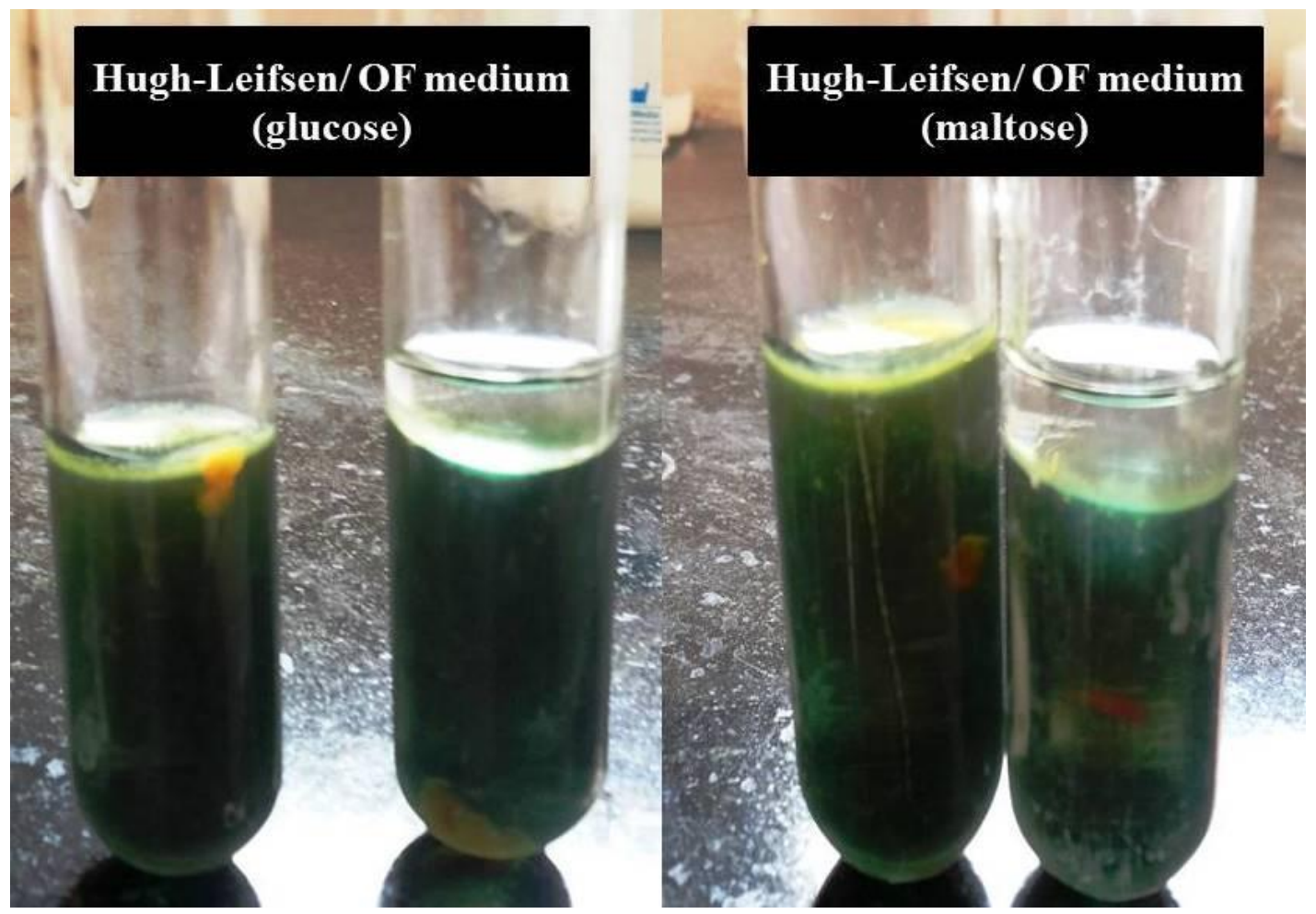


Fig.4 Antibiotic susceptibility testing in Mueller Hinton agar for P.stutzeri(yellowish)- sensitive to imipenem, amikacin, gentamicin, ciprofloxacin, Cefepime, Ceftazidime, Cefotaxime, colistin and resistant to cotrimoxazole.

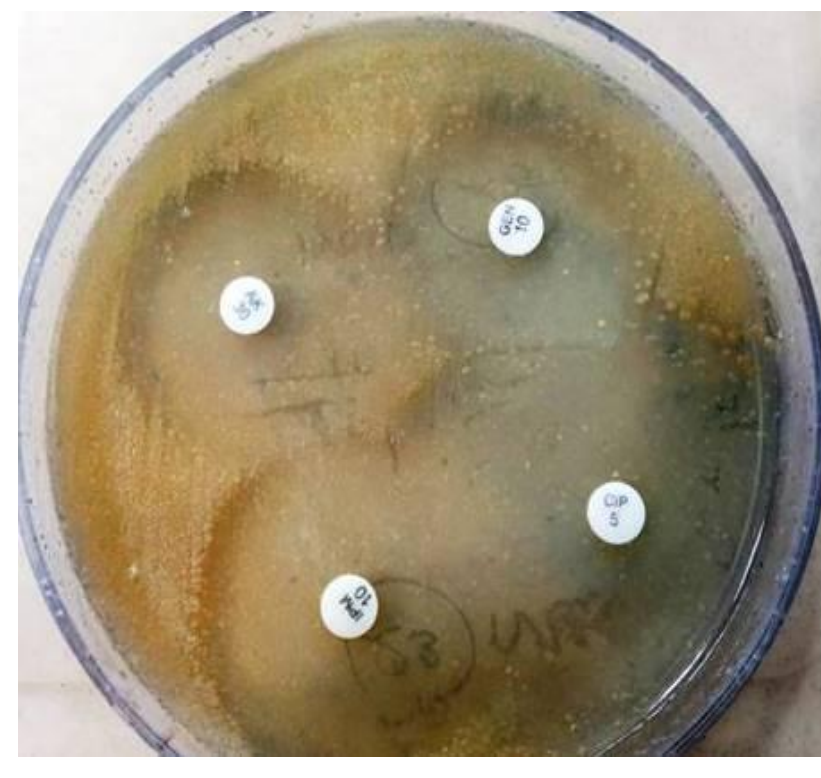

\section{Discussion}

P.Stutzeri is considered as ubiquitous gram negative non-fermenter bacteria in hospital environments. It causes infection in previously sick or immune-suppressed patients and is considered as an opportunistic pathogen. The alarming feature is isolation of this organism from healthy individuals without any risk factors. The genetic transformation is considered as the possibility of acquiring virulence by this organism to infest healthy individuals $(1,2,3)$. P.stutzeri is still infrequently isolated, represents only $1 \%$ of all Pseudomonasisolations, and when it is isolated without significant pathogenicityit is considered as colonization rather than infection (4,5). In 1970's and 1980's, infections with P.stutzeri occurred as an outbreak thatwere attributed to contamination of intravenous fluids, soaps and watersystem used for preparation of skinfor intravenous insertions and haemodialysis respectively $(4,5,6)$. This organism is almost susceptible to most of

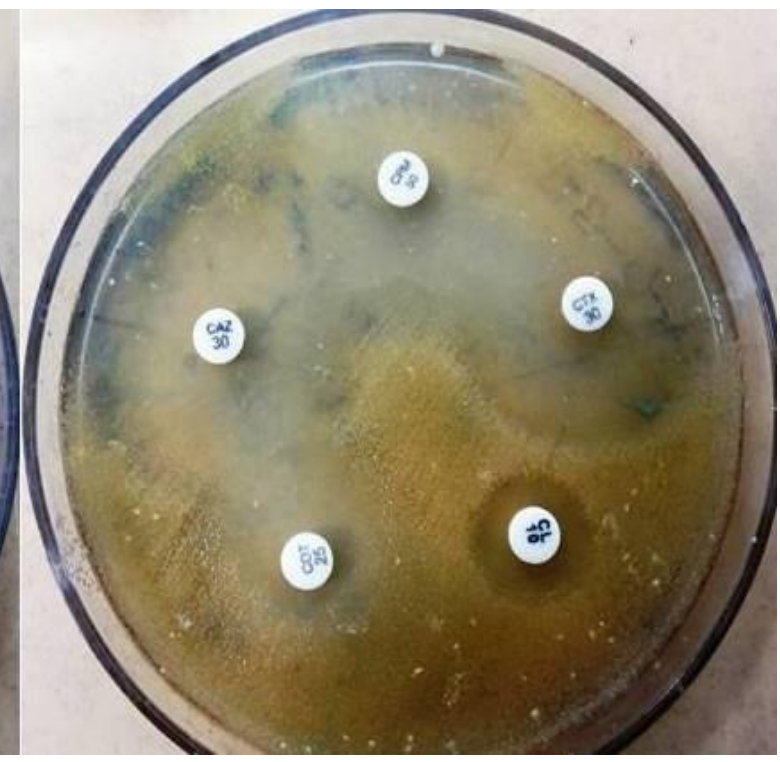

the antibiotics like aminoglycosides, quinolones, Ceftazidime, Cefotaxime, Cefepime, carbapenems (excluding ertapenem), anti-pseudomonal penicillins, polymyxinsand cotrimoxazole $(3,7)$. Resistance in P.stutzeri is acquired by two different mechanisms like alterations in outer membrane proteins and lipopolysaccharide and $\beta$-lactamase production that hydrolyses natural and semi synthetic penicillin $(8,9)$.

\section{Conclusion}

The main aim of this case series is to highlight the dry and wrinkled colonies on blood and MacConkey agar may be neglected as spores especially sputum and other miscellaneous samples. Henceforth dry and wrinkled colonies from clinical samplesmust be processed for gram stain, basic tests like catalase, oxidase, motility, and complete biochemical reactions for speciation and followed by antibiotic susceptibility testing. In general speciation of non-fermenters is important because some of the groups are multi drug resistant 
and intrinsically resistant to many antibiotics like Stenotrophomonas species and Burkholderia species.

\section{References}

1. Gilardi GL. Infrequently encountered Pseudomonas species causing infection in humans. Ann Intern Med. 1972; 77: 211215.

2. Lalucat J, Bennasar A, Bosch R, GarciaValdes E and Palleroni NJ. Biology of Pseudomonas stutzeri. Microbiol MolBiol Rev. 2006; 70: 510-47.

3. Noble RC, Overman SB. Pseudomonas stutzeri infection. A review of hospital isolates and a review of the literature. Diagn Microbiol Infect Dis. 1994; 19: 5156.

4. Keys, T. F., L. J. Melton, M. D. Maker, and D. M.Ilstrup. A suspected hospital outbreak of pseudo bacteremia due to Pseudomonas stutzeri. J. Infect. Dis. 1983; 147:489-493.

5. Goetz, A., Yu VL, Hanchett JE, and Rihs JD.Pseudomonas stutzeri bacteremia associated with hemodialysis. Arch. Intern. Med. 1983; 143:1909-1912.
6. Felts SK, Schaffner W, Melly MA, Koenig MG. Sepsis caused by contaminated intravenous fluids. Epidemiologic, clinical, and laboratory investigation of an outbreak in one hospital. Ann Intern Med. 1972; 77: 88190.

7. Schell RF, Francisco M, Bihl JA, LeFrock JL. The activity of ceftazidime compared with those of aztreonam, newer cephalosporins and Sch 29482 against non-fermentative gram-negative bacilli. Chemotherapy. 1985; 31: 181-90.

8. Tattawasart U, Maillard JY, Furr JR, and Russell AD. Development of resistance to chlorhexidine diacetate and cetylpyridinium chloride in Pseudomonas stutzeri and changes in antibiotic susceptibility. J. Hosp. Infect. 1999; 42:219-229.

9. Franceschini N, Galleni M, Frere JM, Oratore A, and Amicosante G. A class-a beta-lactamase from Pseudomonas stutzeri that is highly active against monobactams and cefotaxime. Biochem. J. 1993; 292:697-700.

\section{How to cite this article:}

Prasanna, S., Sunilkumar Jada and Sathya Raj. 2016. Case Series of Pseudomonas stutzeri at a Tertiary Care Centre from Kancheepuram District, India. Int.J.Curr.Res.Aca.Rev.4(3): 183-188. doi: http://dx.doi.org/10.20546/ijcrar.2016.403.020 